\title{
An assessment of the accuracy of contrast enema for the diagnosis of Hirschsprung disease at a South African tertiary hospital
}

\begin{tabular}{|c|c|}
\hline \multicolumn{2}{|c|}{$\begin{array}{l}\text { Authors: } \\
\text { Monica S. Msomi }{ }^{1} \\
\text { Hansraj Mangray } \\
\text { Vicci du Plessis }\end{array}$} \\
\hline \multicolumn{2}{|c|}{$\begin{array}{l}\text { Affiliations: } \\
{ }^{1} \text { Department of Radiology, } \\
\text { College of Health Sciences, } \\
\text { Nelson R. Mandela School of } \\
\text { Medicine, University of } \\
\text { KwaZulu-Natal, South Africa }\end{array}$} \\
\hline \multicolumn{2}{|c|}{$\begin{array}{l}{ }^{2} \text { Department of Paediatric } \\
\text { Surgery, College of Health } \\
\text { Sciences, Nelson R. Mandela } \\
\text { School of Medicine, } \\
\text { University of KwaZulu-Natal, } \\
\text { South Africa }\end{array}$} \\
\hline \multicolumn{2}{|c|}{$\begin{array}{l}{ }^{3} \text { SKG Radiology, Mandurah, } \\
\text { Australia }\end{array}$} \\
\hline \multicolumn{2}{|c|}{$\begin{array}{l}\text { Corresponding author: } \\
\text { Monica Msomi, } \\
\text { monicasheilamsomi@gmail. } \\
\text { com }\end{array}$} \\
\hline \multicolumn{2}{|c|}{$\begin{array}{l}\text { Received: } 16 \text { Sept. } 2016 \\
\text { Accepted: } 17 \text { Jan. } 2017 \\
\text { Published: } 30 \text { Mar. } 2017\end{array}$} \\
\hline \multicolumn{2}{|c|}{$\begin{array}{l}\text { How to cite this article: } \\
\text { Msomi MS. Mangray H. Du } \\
\text { Plessis V. An assessment of } \\
\text { the accuracy of contrast } \\
\text { enema for the diagnosis of } \\
\text { Hirschsprung disease at a } \\
\text { South African tertiary } \\
\text { hospital. S Afr J Rad. } \\
2017 ; 21(1), \text { a1093. https:// } \\
\text { doi.org/10.4102/sajr. } \\
\text { v21i1.1093 }\end{array}$} \\
\hline \multicolumn{2}{|c|}{$\begin{array}{l}\text { Copyright: } \\
\text { (c) 2017. The Authors. } \\
\text { Licensee: AOSIS. This work } \\
\text { is licensed under the } \\
\text { Creative Commons } \\
\text { Attribution License. }\end{array}$} \\
\hline \multicolumn{2}{|l|}{ Read online: } \\
\hline 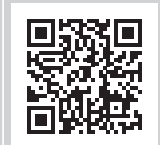 & $\begin{array}{l}\text { Scan this QR } \\
\text { code with your } \\
\text { smart phone or } \\
\text { mobile device } \\
\text { to read online. }\end{array}$ \\
\hline
\end{tabular}

Objectives: To compare radiological findings with the histological diagnosis of Hirschsprung disease (HD) to establish the usefulness of contrast enema as an initial screening and diagnostic tool. To correlate accuracy of radiological diagnosis at Grey's Hospital with international standards.

Materials and methods: Systematic searches were conducted through the Picture Archiving and Communication System and the National Health Laboratory Service records for patients aged 0-12 years, with clinically suspected HD, for whom both contrast enemas and rectal biopsies were performed between 01 January 2011 and 31 August 2015 in a tertiary-level hospital. A total of 54 such patients were identified. Diagnostic accuracy levels were calculated by comparing radiological results with histology results, which is the gold standard.

Results: Diagnostic accuracy of contrast enema was $78 \%$, sensitivity was $94.4 \%$ and the negative predictive value was $95.7 \%$. Specificity $(68.8 \%)$ and positive predictive values (63\%) were considerably lower. A lower false-negative rate of $5.6 \%$ was obtained at Grey's Hospital as compared with the international reports of up to $30 \%$.

Conclusion: Contrast enema remains useful as an initial screening and diagnostic test for HD. Results of this South African tertiary referral hospital were consistent with the best international results for sensitivity of the contrast enema (approximately $80 \%-88 \%$ in excluding the disease).

\section{Introduction}

Hirschsprung disease (HD), also known as colonic aganglionosis, is a rare cause of constipation. It is defined as a functional obstruction of bowel caused by the lack of distal enteric ganglion cells with a reported incidence of 1:5000-7200 in newborns. ${ }^{1}$ Variable lengths and segments of bowel may be involved. It is associated with significant morbidity if not diagnosed and treated early; therefore, clinicians are often cautious to promptly identify or exclude this disease. There has been considerable debate about the most appropriate initial test to diagnose HD because all the diagnostic modalities [contrast enema, anorectal manometry (ARM) and rectal suction biopsy] can produce false-negative (FN) and false-positive (FP) results. ${ }^{2}$ Following critical evaluation of the sensitivity, specificity and predictive values of these investigations in the 1980s, it was shown that radiologic studies alone are not a sensitive enough tool to exclude HD and that manometry and rectal mucosal biopsy are also required for an accurate diagnosis. ${ }^{3}$ Full-thickness biopsy confirming the absence of ganglion cells remains the gold standard for diagnosis. ${ }^{4}$ However, the risks associated with rectal biopsy, including bleeding, sepsis, perforation and anaesthesia-related risks, ${ }^{5}$ led to the development of the rectal suction biopsy as an alternative diagnostic modality, but as mentioned previously, it can produce FP and FN results. Contrast enemas therefore continue to play an invaluable role in many centres, and a radiologist's opinion forms part of the data used by clinicians to decide whether other investigations are necessary in neonates and children with distal bowel obstruction or abnormal bowel habits. ${ }^{3}$ The contrast enema is often readily available and is the first imaging procedure performed in many centres, including Grey's Hospital, a tertiary referral hospital in Pietermaritzburg, South Africa. However, much controversy exists in the literature regarding its usefulness in the work up of patients with HD. ${ }^{4,6}$ The best reported sensitivity of the barium enema in excluding HD is approximately $80 \%-88 \%{ }^{6}$ and FN rates of up to $30 \%$ have been reported. ${ }^{4}$ The diagnostic accuracy of the contrast enema is, therefore, of continued interest to both clinicians and radiologists, and it is important to constantly assess how accurately radiologists are able to make the diagnosis of $\mathrm{HD}$, with a view to both encouraging good radiological practice as well as identifying possible factors that may contribute to reduced diagnostic accuracy. 


\section{Methods \\ Study sample and design}

A retrospective audit was undertaken of all contrast enemas and rectal biopsies performed on patients aged 0-12 years between 01 January 2011 and 31 August 2015 inclusive. Only children investigated with both contrast enema and fullthickness rectal biopsy for the clinical suspicion of HD were included in the study.

\section{Study description}

A systematic search was conducted through the Picture Archiving and Communication System (PACS) for patients aged 0-12 years who had been referred for contrast enemas between 01 January 2011 and 31 August 2015 inclusive. A total of 185 patients were identified. The request forms for these patients were reviewed to identify the patients who were specifically referred for the diagnosis or exclusion of HD (history of constipation or changed bowel habits). The total number of patients identified was 97. A concurrent search for rectal biopsy results was conducted through the National Health Laboratory Service (NHLS) website, and ultimately a total of 54 patients were identified who had undergone both contrast enema and biopsy. The remaining 43 patients were excluded from the study because they did not meet the selection criteria and were not investigated further for the purposes of our study.

Enemas were performed at our tertiary institution mostly by trainee radiologists and occasionally by specialist radiologists, while the images were predominantly examined by the performing trainee radiologist in conjunction with a specialist radiologist; on a few occasions, images were examined by a radiology registrar alone. Only water-soluble contrast enemas (WSCEs) are performed at Grey's Hospital using gastrografin, omnipaque or ultravist as contrast agents. For the procedure, a Foley's catheter is inserted into the lower rectum and the catheter bulb is not inflated. Contrast is passed as far as possible, in most cases until the terminal ileum is opacified. For our study, positive enema results were based mainly on the presence of a transition zone (TZ) or calibre change, reversed recto-sigmoid ratio (RRSR) and other ancillary findings such as delayed post-contrast evacuation of bowel, saw-tooth mucosal pattern or mucosal irregularity.

Full-thickness rectal biopsies were performed under general anaesthesia by a specialist paediatric surgeon at $15 \mathrm{~mm}$ above the dentate line, posteriorly, and sent for haematoxylin and eosin (H\&E) staining and, occasionally, for calretinin staining. (Suction biopsy equipment was not available during the study period but has since been acquired.) Specimens were examined by a specialist histopathologist, who looked for the presence or absence of ganglion cells in Meissner's submucosal plexus and Auerbach's intermyenteric plexus, at times with the additional finding of neuronal hyperplasia.

Related symptoms such as constipation, abdominal distension, vomiting and delayed passage of meconium (where present) were recorded for each patient to ascertain any statistical significance.

A data sheet was used to collect the relevant data. The study was approved by the Biomedical Research Ethics Committee (BE475/15), the Provincial Department of Health and the management of Grey's Hospital.

\section{Ethical consideration}

This is a blind, retrospective, descriptive study, focusing on the analysis of radiological and histopathological records. Patients' identities are protected and no consent was necessary.

\section{Statistical analysis}

Data were collected and entered into a computerised database program (Microsoft Excel). A codebook which included variable names, descriptions and formats was developed. Sensitivity and specificity rates for contrast enemas within 95\% CIs (confidence intervals) were calculated with the method of Wilson. The single inconclusive enema result was excluded from this calculation. Risk factors associated with a positive histology result were identified using Fisher's exact test. Factors associated with a significant $p$-value at the univariate level were included in a multivariable logistic model. Because of the relatively small number of positive cases, each risk factor was also modelled separately adjusting for age and sex. A two-sided $p$-value of $<0.05$ was considered statistically significant for all analyses. Data were analysed using the Stata v13.1 statistical software.

\section{Results}

Of the 54 patients included in the study, 28 had positive contrast enemas, 25 had negative enemas and 1 had an inconclusive enema result (Table 1). Seventeen patients who had positive enemas also had positive histology [truepositives (TPs)], while for the remaining 11 patients with positive enemas, 10 patients turned out to have negative histology (FP) and 1 had an inconclusive histology result. Of the 25 negative enemas, most (22) had concurrent negative histology (true-negatives), one had positive histology (FN) and two had inconclusive histology results. The single inconclusive enema result had a negative histology result. The three inconclusive histology results were as a result of inadequate or non-representative samples. The inconclusive enema result was because of marked distension of the rectum with faeces and no other supporting features of HD.

Table 2 denotes the diagnostic evaluation of the contrast enema compared with rectal biopsy as the gold standard.

TABLE 1: Screening test evaluation: Single table analysis.

\begin{tabular}{lccc}
\hline $\begin{array}{l}\text { Contrast enema } \\
\text { result }\end{array}$ & \multicolumn{3}{c}{ Histology result } \\
\cline { 2 - 4 } & Positive & Negative & Total \\
\hline Positive & 17 & 10 & 27 \\
Negative & 1 & 22 & 23 \\
\hline Total & 18 & 32 & 50 \\
\hline
\end{tabular}


TABLE 2: Diagnostic evaluation of the contrast enema compared to rectal biopsy as the gold standard.

\begin{tabular}{lcc}
\hline Parameter & Estimated \% & 95\% confidence interval \\
\hline Sensitivity & 94.4 & $74.2-99.0$ \\
Specificity & 68.8 & $51.4-82.1$ \\
Positive predictive value & 63.0 & $44.2-78.5$ \\
Negative predictive value & 95.7 & $79.0-99.2$ \\
Diagnostic accuracy & 78.0 & $64.8-87.3$ \\
\hline
\end{tabular}

TABLE 3: Risk factors for the disease adjusted for age and sex.

\begin{tabular}{lccc}
\hline Risk factor & Odds ratio & $\boldsymbol{p}^{*}$ & $\begin{array}{c}\mathbf{9 5 \%} \text { confidence } \\
\text { interval }\end{array}$ \\
\hline $\begin{array}{l}\text { Demographics } \\
\text { Age }<1 \text { month }\end{array}$ & 6.9 & 0.07 & $0.9-54.5$ \\
$\quad$ Male sex & 9.3 & 0.05 & $1.1-82.7$ \\
Radiological features $\dagger$ & & & \\
$\quad$ Transition zone & 4.5 & 0.07 & $09-22.3$ \\
$\quad$ Reversed recto-sigmoid ratio & 3.4 & 0.2 & $0.6-18.4$ \\
\hline
\end{tabular}

*, all values as calculated by chi-square and Fisher's exact tests.

$\dagger$, The transition zone and reversed recto-sigmoid ratio were the only radiological features that were statistically significant after adjusting for age and sex. None of the symptoms were statistically significant when adjusted for age and sex. Although vomiting was significantly statistically significant when adjusted for age and sex. Although vomiting was significantly asociatically significant when adjusted for age and sex [OR: $3.6 ; 95 \% \mathrm{Cl} 0.7-18.7 ; p=0.1]$.

In terms of risk factors for the disease, with adjustments for age and sex, children who were less than 1 month had a greater chance of testing positive on histology for the disease than children over 1 month of age (Table 3). Males also had a significantly greater chance of testing positive than females. In terms of radiological features, the presence of an RRSR and a TZ each significantly increased the chances of testing positive for the disease (OR 3.4, 95\% CI 0.6-18.4, $p=0.2$ for RRSR and OR 4.5, 95\% CI 0.9-22.3, $p=0.07$ for TZ, respectively) (Table 3 ). Of the related symptoms, only vomiting was significantly associated with the disease (OR 3.7, 95\% CI 1.1-12.9, $p=0.03$ ); however, this was not statistically significant when adjusted for age and sex (OR $3.6,95 \%$ CI $0.7-18.7, p=0.1)$.

\section{Discussion}

Results of the Grey's Hospital were comparable with the best international results for sensitivity of the contrast enema (approximately 80\% - 88\%) for excluding HD. ${ }^{6}$ There is, however, a wide discrepancy between sensitivity and specificity rates. A systematic review by de Lorijn et al. concluded that such a discrepancy may be due to differences in the quality of studies and variation as a result of chance because of the low number of included patients. ${ }^{2}$ Nevertheless, lower specificity rates are desirable for screening tests as the benefits of over-referring patients for biopsies, early diagnosis and management of the disease outweigh the risks and costs of missing patients.

The high negative predictive value (NPV) of 95.7\% was consistent with those reported in literature and suggests that very few positive cases are missed, which supports the usefulness of the contrast enema. ${ }^{6}$ A lower FN rate of $5.6 \%$ was obtained as compared to the international reports of up to $30 \%$. This may be because this condition is seen significantly more frequently at the Grey's Hospital as compared to other institutions. Seventeen out of the 54 studied patients had biopsy-proven HD; this number is significantly higher than the accepted prevalence of the disease and may be related to the fact that the study was conducted in a tertiary-care hospital. The sole FN result was in a child with cerebral palsy and may be related to regular rectal washouts or digital rectal manipulation, which is often performed on cerebral palsy patients and have been shown to lead to FN results., ${ }^{2,4}$ The significantly higher FP rate of $31.3 \%$, although concerning for radiological overdiagnosis, which may lead to unnecessary biopsies, is desirable for screening tests such as contrast enemas for the aforementioned reasons.

A study by O'Donovan et al. assessed the validity of using low osmolality WSCE instead of barium enemas in neonates and infants for the investigation of HD. ${ }^{3}$ It found good agreement in the sensitivities and specificities for both groups, which justifies the use of WSCE in neonates and infants. The RRSR and the TZ were the only radiological features that were significantly associated with the disease. The RRSR was even more significantly associated with the disease than the TZ. An RRSR was present in $72.2 \%$ of children with the disease and in only $27.3 \%$ of children without the disease. The TZ was present in $66.7 \%$ of children with the disease and in only $18.2 \%$ of children without the disease. This is consistent with most literature reports which state that the presence of a radiographic TZ on barium enema remains the most accurate diagnostic sign for $\mathrm{HD}, 2,7,8,9$ and with the study by $\mathrm{O}^{\prime}$ Donovan et al., ${ }^{3}$ which reported the RRSR as the most sensitive radiologic sign of HD (Figures 1-3). The radiographic TZ is also known to be the most reliable radiological sign to evaluate the level of aganglionosis, a finding that has implications for surgical management. ${ }^{9}$ Notwithstanding these factors, Taxman et al. suggested that in young infants, the calibre change or TZ is more difficult to demonstrate. ${ }^{4}$ Furthermore, De Campo et al. demonstrated a normal calibre colon in $75 \%$ of children with total aganglionosis, ${ }^{2}$ which means that the absence of this feature on contrast enema does not exclude HD.

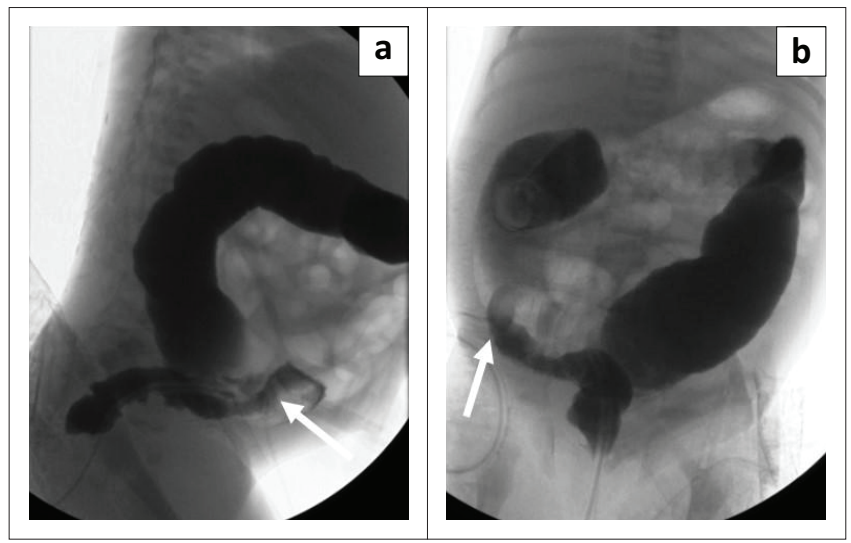

FIGURE 1: Lateral (a) and frontal (b) views demonstrating long segment Hirschsprung disease involving the rectum and distal sigmoid colon, with a transition zone (white arrows) seen in the sigmoid colon. 

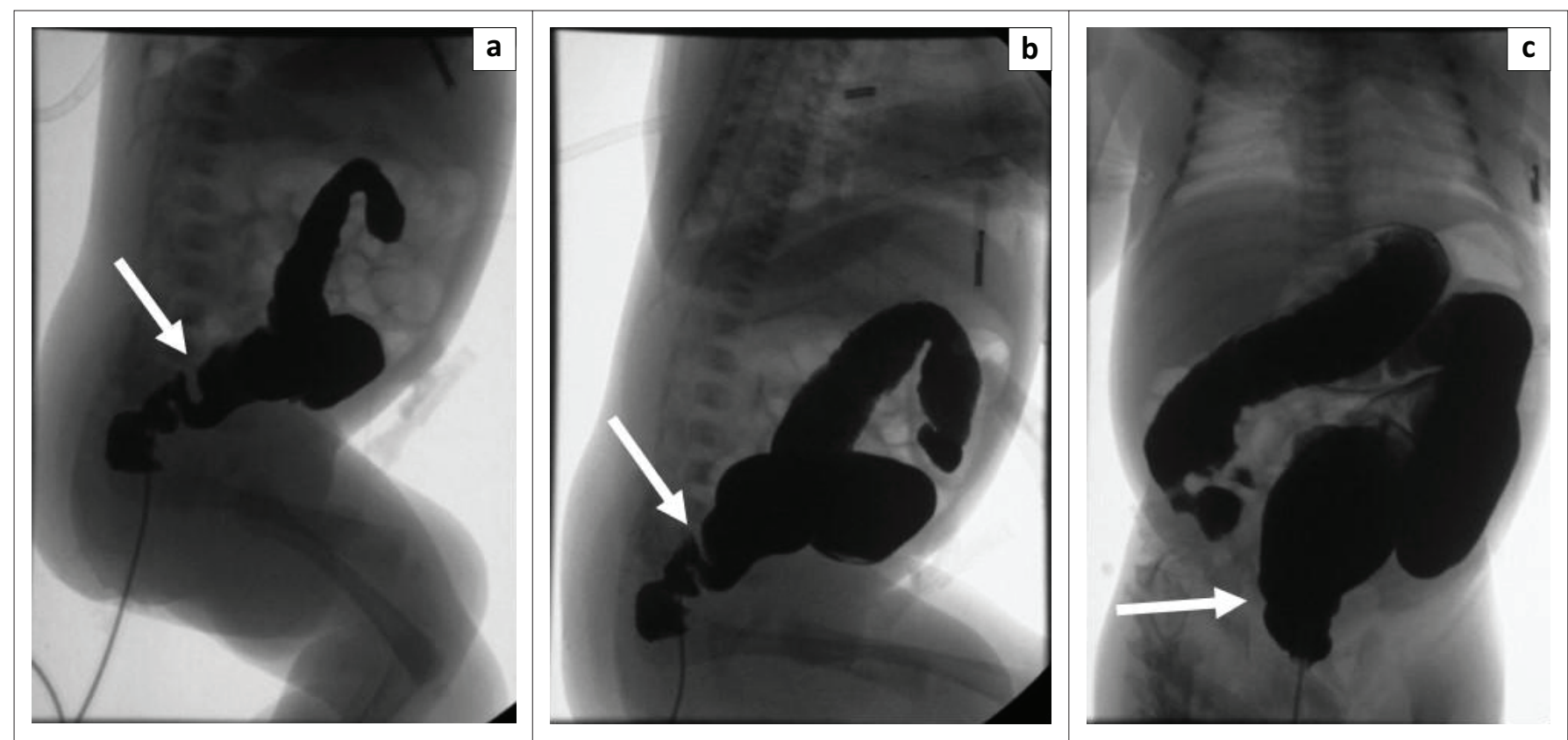

FIGURE 2: Lateral (a), oblique (b) and frontal (c) views demonstrating a zone of transition in the region of the recto-sigmoid junction (white arrows) in a patient with Hirschsprung disease. A reversed recto-sigmoid ratio is also seen.

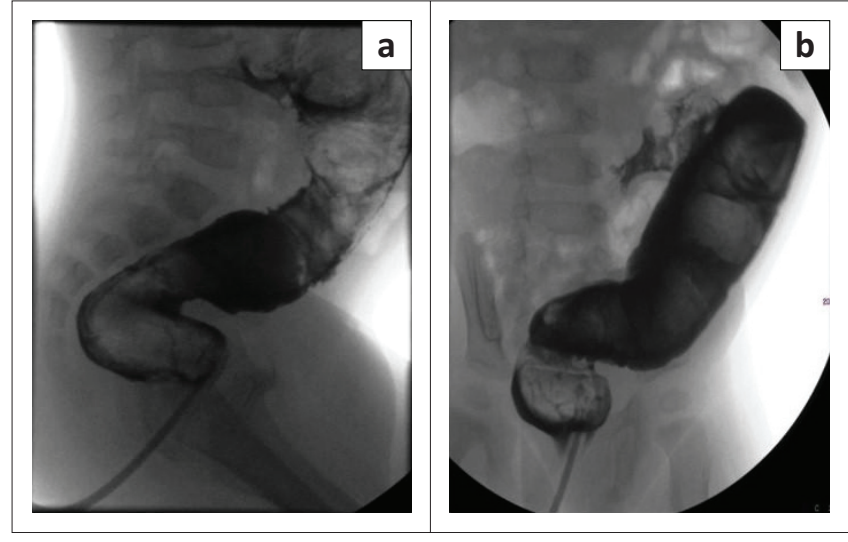

FIGURE 3: Lateral (a) and frontal (b) views in a patient with Hirschsprung disease. The lateral view demonstrates a reversed recto-sigmoid ratio.

In this study, we did not assess for any correlation between the TZs at contrast enema and at surgery.

The most common presenting symptoms for HD are delayed passage of meconium $(60 \%-90 \%)$, abdominal distension $(63 \%-91 \%)$ and bilious vomiting $(19 \%-37 \%) .{ }^{1}$ In older children, the most frequently reported symptom is constipation. ${ }^{1}$ Our study however showed that for all age groups, vomiting was the only symptom that was significantly associated with the disease, although this was not statistically significant when adjusted for age and sex, as aforementioned. Delayed passage of meconium (beyond 24h) was documented in only one child, and while constipation and abdominal distension were reported in patients with and without the disease, no statistical significance was established with these symptoms. Lewis et al. demonstrated that patients with HD were more likely to experience symptoms within the first week of life and have delayed passage of meconium, abdominal distension, vomiting and a TZ. ${ }^{10}$ Our study was in concordance with at least three of these findings.
Most of our patients with HD were identified in the neonatal period, had the symptom of vomiting and had a TZ on contrast enema. Our findings of neonatal age and male sex as risk factors for the disease were also consistent with literature reports. Ninety per cent of patients are diagnosed when they are newborns, and the disease is reported to be four times more common in males than females. ${ }^{1}$

Diamond et al. reported that on a univariate analysis, age below 30 days, female sex, absence of a TZ and presence of another finding were all statistically, at the 0.05 level, associated with an increased risk of an FP contrast enema result. The presence of bilious emesis or the passage of meconium beyond the first day of life was associated with a decreased risk of an FP result. ${ }^{10}$ The same study also found that on multivariate analyses, the absence of a TZ, female sex and age below 30 days were all associated with an increased risk of an FP result, whereas a history of bilious emesis decreased the risk.

Our study showed that females are more than three times as likely to be FP as compared to males; $30 \%$ of males are FP versus $60 \%$ of females (OR $3.4,95 \%$ CI $0.5-25, p=0.2$ ). This is consistent with the finding of the report by Diamond et al., but the figures are not statistically significant. Absence of a $\mathrm{TZ}$ was found more often in FP (40\%) than TP (33\%) patients, although again no statistical association was found. Vomiting was found more often in TP rather than in FP patients (OR 2.3, 95\% CI 0.5-12, $p=0.4$ ). Forty-four per cent of children without vomiting were FP versus $25 \%$ of children with vomiting (OR $0.4,95 \%$ CI $0.1-2.2, p=0.3$ ). The low OR suggests a lower chance of FP with vomiting and provides some support to the findings of the report. Our study showed no significant association between another radiological finding and an FP enema result. 
According to our study, age above 30 days is an indication of an FP enema result, which contradicts the finding of the report by Diamond et al. There was only one reported case of delayed passage of meconium in our study, so no comparison could be made concerning this feature.

Factors associated with a TP result in our study include age less than 30 days, male sex and children presenting with vomiting (Table 4). No statistical significance was demonstrated with any of these factors, but the high OR values (OR 7.2, 95\% CI 0.7-69.4, $p=0.1$; OR 3.4, 95\% CI 0.5-25.3, $p=0.3$ and OR $2.3,95 \%$ CI $0.5-12, p=0.4$, respectively) suggest an association.

Lastly, a note about ARM. This is a non-invasive diagnostic test, easy to perform in children older than 1 year and has often been suggested as an ideal screening tool. ${ }^{2}$ A study by de Lorijn et al. concluded that it is slightly more sensitive ( $83 \%$ vs. $76 \%$ ), although less specific ( $93 \%$ vs. $97 \%$ ) than contrast enemas. ${ }^{4}$ Unfortunately, the costs involved preclude its implementation in our institution.

\section{Limitations}

The study is limited to a certain age group. The correct histopathological diagnosis is dependent on the technique of rectal biopsy. Radiological interpretation is dependent on the degree of experience of the interpreting radiologist, as well as the study technique and quality of images, which are in turn influenced by the degree of experience of the performing radiologists who in certain instances were junior trainees. Patient factors such as lack of co-operation as can be seen with younger patients may also influence the quality of the study or images. The sample size is reduced.

TABLE 4: Risk factors associated with true-positives

\begin{tabular}{lccc}
\hline Risk factor & Odds ratio & $p$-value* & $\begin{array}{c}95 \% \text { confidence } \\
\text { interval }\end{array}$ \\
\hline Demographics & 7.2 & 0.1 & $0.7-69.4$ \\
Age $<1$ month & 3.4 & 0.3 & $0.5-25.3$ \\
Male sex & & & \\
Radiological features & 1.3 & 0.9 & $0.3-6.6$ \\
Transition zone & 0.3 & 0.4 & $0.03-2.9$ \\
Reversed Recto-Sigmoid ratio & 1.2 & 0.9 & $0.2-6.2$ \\
Other & $\mathrm{n} / \mathrm{a}$ & 0.9 & $\mathrm{n} / \mathrm{a}$ \\
Unrelated & & & \\
Symptoms & 2.3 & 0.4 & $0.5-12.0$ \\
Vomiting & 1.3 & 0.9 & $0.3-5.9$ \\
Constipation & 1.0 & 0.9 & $0.2-4.7$ \\
Abdominal distention & $\mathrm{n} / \mathrm{a}$ & 0.9 & $\mathrm{n} / \mathrm{a}$ \\
Delayed passage of Meconium & $\mathrm{n} / \mathrm{a}$ & 0.5 & $\mathrm{n} / \mathrm{a}$ \\
\hline Dysmorphic features & &
\end{tabular}

*, all values as calculated by Fisher's exact test.

Other, delayed post-contrast evaluation of bowel, serrated outline if lower mucosal wall, saw-tooth mucosal pattern, mucosal irregularity, corrugated convoluted rectum and sigmoid; Unrelated, features not related to Hirschsprung disease; n/a, not applicable (cannot be calculated due to 0 false-positive).

Note: None of the values are statistically significant, but the high OR associated with patients aged less than one month (neonates), male patients and with vomiting suggest a strong association with true positive finding.

\section{Conclusion}

The contrast enema remains useful as a screening test for HD. Results of this South African tertiary referral hospital were consistent with the best international results for sensitivity of the contrast enema (approximately $80 \%-88 \%$ in excluding the disease). However, full-thickness rectal biopsy remains the gold standard for diagnosis of HD.

\section{Acknowledgements}

The authors would like to thank Catherine Connolly for her assistance with data analysis and Dr Matthew Goodier for his assistance with data analysis and suggested revisions for this article.

\section{Competing interests}

The authors declare that they have no financial or personal relationships that may have inappropriately influenced them in writing this article.

\section{Authors' contributions}

M.S.M. performed literature review, prepared research proposal protocol for ethics board approval, gathered data for analysis, and prepared the primary write-up of the research manuscript. H.M. was the co-investigator of the research project, assisted with data collection for analysis and helped with manuscript editing. V.d.P. supervised the research project and helped with the manuscript editing.

\section{References}

1. Merrow C. Hirschsprung disease [homepage on the Internet]. 2011 [cited 2015 Sep 29]. Available from: http://my.statdx.com/Document/Hirschsprung-Disease/ 6b6f4318-Afc8-4809-Aaf7-1c5a001ca889

2. de Lorijn F, Kremer LC, Reitsma JB, Benninga MA. Diagnostic tests in Hirschsprung disease: A systematic review. J Pediatr Gastroenterol Nutr. 2006;42(5):496-505. https://doi.org/10.1097/01.mpg.0000214164.90939.92

3. O'Donovan AN, Habra G, Somers S, Malone DE, Rees A, Winthrop AL. Diagnosis of Hirschsprung's disease. Am J Roentgenol. 1996;167(2):517-520. https://doi. org/10.2214/ajr.167.2.8686640

4. De Lorijn F, Reitsma JB, Voskuijl WP, et al. Diagnosis of Hirschsprung's disease: A prospective, comparative accuracy study of common tests. I Pediatr. 2005;146(6):787-792. https://doi.org/10.1016/j.jpeds.2005.01.044

5. Alehossein M, Roohi A, Pourgholami M, Mollaeian M, Salamati P. Diagnostic accuracy of radiologic scoring system for evaluation of suspicious Hirschsprung
disease in children. Iran J Radiol. 2015;12(2):e12451. https://doi.org/10.5812/ iranjradiol.12451

6. Reid JR, Buonomo C, Moreira C, Kozakevich H, Nurko SJ. The barium enema in constipation: Comparison with rectal manometry and biopsy to exclude Hirschsprung's disease after the neonatal period. Pediatr Radiol. 2000;30(10):681684. https://doi.org/10.1007/s002470000298
680

7. Proctor ML, Traubici J, Langer JC, et al. Correlation between radiographic transition zone and level of aganglionosis in Hirschsprung's disease: Implications for surgical approach. J Pediatr Surg. 2003;38(5):775-778. https://doi.org/10.1016/jpsu. 2003.50165

8. Taxman TL, Yulish BS, Rothstein FC. How useful is the barium enema in the diagnosis of infantile Hirschsprung's disease? Am J Dis Child. 1986;140(9):881-884.

9. Rosenfield NS, Ablow RC, Markowitz RI, et al. Hirschsprung disease: Accuracy of the barium enema examination. Radiology. 1984;150(2):393-400. https://doi. org/10.1148/radiology.150.2.6691093

10. Diamond IR, Casadiego G, Traubici J, Langer JC, Wales PW. The contrast enema for Hirschsprung disease: Predictors of a false-positive result. J Pediatr Surg. 2007;42(5):792-795. https://doi.org/10.1016/j.jpedsurg.2006.12.031 